\title{
Acute and 26-Week Repeated Oral Dose Toxicity Study of UP446, a Combination of Scutellaria Extract and Acacia Extract in Rats
}

\author{
Young Chul Lee ${ }^{1}$, Eujin Hyun ${ }^{1}$, Mesfin Yimam², Lidia Brownell ${ }^{2}$, Qi Jia ${ }^{2}$ \\ ${ }^{1}$ Unigen Inc., Cheonan, South Korea; ${ }^{2}$ Unigen Inc., Seattle, USA. \\ Email: yclee@unigen.net, QJia@unigen.net
}

Received March 21 $1^{\text {st }}, 2013$; revised April 22 ${ }^{\text {nd }}, 2013$; accepted April 30 $0^{\text {th }}, 2013$

Copyright (C) 2013 Young Chul Lee et al. This is an open access article distributed under the Creative Commons Attribution License, which permits unrestricted use, distribution, and reproduction in any medium, provided the original work is properly cited.

\begin{abstract}
UP446 has been used in both joint supplements and prescription medical food. The purpose of this study was to evaluate the pharmaceutical safety of UP446 via acute and 26-week repeated oral dose toxicity study in SD rats. In acute toxicity study, UP446 was administered by oral gavage to Sprague-Dawley rats ( 5 males and 5 females) at a dose of 5000 $\mathrm{mg} / \mathrm{kg}$. In 26-week repeated oral dose toxicity study, UP446 at doses of 500,1000 and $2000 \mathrm{mg} / \mathrm{kg} /$ day were given orally to groups of rats (10 rats/dose/sex) for 26 -week. UP446 at a dose of $5000 \mathrm{mg} / \mathrm{kg}$ produced no treatment-related acute toxicity or mortality in any of the animals tested during 14 days of the study. In 26-week repeated dose toxicity study, there was no significant difference in body weight between the control and all treatment groups. Blackish stool and soft stool was observed in one male in the $1000 \mathrm{mg} / \mathrm{kg}$ group and in some males and females of $2000 \mathrm{mg} / \mathrm{kg}$ group. However, these changes of stool were not considered to be toxic effects because neither histopathological change in gastrointestinal tracks (GIT) nor body weight change were detected. No drug induced abnormalities were found as of body weights, food consumption, ophthalmological examinations, urinalysis, hematology, clinical chemistry, organ weights and gross necropsy in any animals in the dosing groups. These results suggest that the oral lethal dose of UP446 for male and female rats is in excess of $5000 \mathrm{mg} / \mathrm{kg}$ and the no observed adverse effect level (NOAEL) of the UP446 for both male and female rats is considered to be greater than $2000 \mathrm{mg} / \mathrm{kg} / \mathrm{day}$.
\end{abstract}

Keywords: Scutellaria baicalensis; Acacia catechu; Acute Oral Toxicity; Repeated Oral Toxicity

\section{Introduction}

The formulations containing multiple plant extracts have attained wide recognition in comparison to crude plant materials and extracts, due to reduction in dose, convenience, and ease of administration. These formulations are used by large sections of the human population particularly in developed countries. Many synthetic drugs are known to act on a single molecular target but the multitarget responses of herbal formulations are proven to be beneficial in chronic conditions [1-3]. The World Health Organization (WHO) insists that the safety of herbal medicines is a critical component in the quality control of healthcare products. However, lack of adequate regulations, the pharmacological complexity of herbal products, and the insufficiency of information on the pharmacology and toxicity of these compounds are sometimes becoming medical issues, even though the side effects are minimal $[4,5]$.
Thus a crucial standardization and toxicological evaluation of the safety in pre-clinical model could be relevant. Flavonoids are polyphenolic compounds that are ubiquitous in nature and are categorized, according to chemical structure, into flavonols, flavones, flavanones, isoflavones, catechins, anthocyanidins, and chalcones. Over 4000 flavonoids have been identified, many of which present in daily consumed human foods such as fruits, vegetables, and beverages including tea, coffee, beer, wine, and fruit drinks as well as traditional medicines and pharmaceutical drugs [6]. Usage of flavonoids include food flavor, fruits and vegetables pigments, antimicrobial, antiviral, antioxidants [7-9], as well as anticarcinogenic and antimutagenic [10]. Primarily two bioflavonoids, baicalin from Scutellaria baicalensis (S. baicalensis) and catechin from Acacia catechu (A. catechu) have been used separately in many traditional medicines and pharmaceutical products for a variety of uses including anti-inflamma- 
tory, antiviral, antibacterial, anticancer, and cardiovascular applications [7,11-17].

UP446 is a standardized bioflavonoid composition with primarily baicalin from the roots of Scutellaria baicalensis (S. baicalensis) and (+)-catechin from the heartwoods of Acacia catechu (A. catechu) [18]. It has been used in both over the counter joint care dietary supplements and prescription medical food [19]. The therapeutic dosage of UP446 is $250-500 \mathrm{mg}$ per day in clinical applications [20]. Previous studies have shown that UP446 reduces production of eicosanoids through inhibition of cyclooxygenase-1 (COX-1), cyclooxygenase-2 (COX-2), and 5-lipoxygenase (5-LOX) enzymes and also decreases expressions of inducible nitric oxide synthase (iNOS), nuclear factor-kappaB (NF-kB), and tumor necrosis factor-alpha (TNF- $\alpha$ ) [21]. Furthermore, UP446 showed analgesic effects on multiple in vivo models [22].

The safety of UP446 was partially evaluated in 90 days repeated oral toxicity study in rats [23] but long term assessment for toxicity in rats is required because most patients with chronic arthritis diseases undergo long term therapy.

The objective of the current study is to provide a comprehensive supplement to the safety profile reported previously for a standardized composition of $S$. baicalensis and $A$. catechu. Herein, two toxicity studies were performed to evaluate the safety of UP446. The acute oral toxicity study was carried out at a high dose, whereas the 26-week repeated oral toxicity study was performed to establish the no-observed-adverse-effect level (NOAEL) of UP446.

\section{Materials and Methods}

\subsection{Preparation of UP446}

Detailed method for preparation of the two major flavonoids, baicalin and catechin, from the roots of $S$. baicalensis and the heartwoods of $A$. catechu, respectively, were disclosed in a US patent [24]. Briefly, S. baicalensis extract from roots was extracted with water and then recrystallized. The $S$. baicalensis extract contained baicalin as the major bioflavonoid at content not less than $75 \%$ as well as other minor free-B-ring flavonoids such as wogonin-7-O-G-glucuronide, oroxylin A-7-OG-glucuronide, and baicalein. Catechin extract was obtained from repeated crystallization of an aqueous extraction of the heartwoods of an India medicinal plant, A. catechu. (+)Catechin is the major component in the A. catechu extract with a content of not less than $65 \%$ plus a minor amount of its enantiomer, epicatechin, as well as other minor amounts of flavans. Analyses of the extracts were performed separately by two high-performance liquid chromatography (HPLC) methods. The quantification re- sults of baicalin, from the $S$. baicalensis extract and catechin from the $A$. catech $u$ extract were calculated by comparison HPLC peak area with known standards. The final UP446 composition was a mixture of $S$. baicalensis and $A$. catech $u$ standardized extracts at a ratio $4: 1$ with baicalin content not less than $60 \%$ and catechin content not less than $10 \%$. Other minor flavonoids, such as wogonin 7 -glucuronide and baicalein, account for about $15 \%$ of total weight. Moisture, ash, fat, and fiber constitute the remainder weight (Figure 1).

\subsection{Experimental Animals}

Male and female Sprague-Dawley rats, aged 5 weeks, were purchased from the ORIENTBIO INC. in Korea. In the acute oral toxicity study, 10 rats of each sex were used, whereas in the 26-week repeated oral dose toxicity study, 50 rats of each sex including recovery groups were used. All rats were labeled by tailmark and housed singly in stainless wire mesh cages in a room maintained under environmentally controlled conditions of $21^{\circ} \mathrm{C} \pm 2^{\circ} \mathrm{C}$ and a $12 \mathrm{~h}$ light-dark cycle in the animal facility of Biotoxtech Co., Ltd. All rats were acclimatized at least one week before starting the experiments, and had free access to water and food. Test article was mixed in a solution of $0.5 \%$ carboxymethylcellulose sodium salt (CMC$\mathrm{Na}$ ) in water (Choongwae Pharma Corp). The control group received the vehicle only at the same volume as the test animals. Clinical signs were observed in all animals during the experiments.

\subsection{Acute Oral Toxicity Study}

This study was performed at the Biotoxtech GLP institute following approval of the Institutional Animal Care and Use Committee (Approval No.: 110947 and Study No.: B11996) based on Animal Protection Act and conducted in compliance with the Organization for Economic Cooperation and Development (OECD) Guideline 425 adopted on the 3rd of October 2008 and Korea Food and Drug Administration (KFDA) Guideline.

After acclimatization, 10 rats of each sex were randomly divided into two groups of 5 males and 5 females and were treated by gavage at doses of 0 and $5000 \mathrm{mg} / \mathrm{kg}$ body weight. Mortality, clinical signs, body weight changes and gross findings were monitored during the 14 days after treatment.

\subsection{6-Week Repeated Oral Dose Toxicity Study}

This study was performed at the Biotoxtech GLP institute following approval of the Institutional Animal Care and Use Committee (Approval No.: 110736 and Study No.: B11287) and conducted in compliance with the Organization for Economic Cooperation and Development 


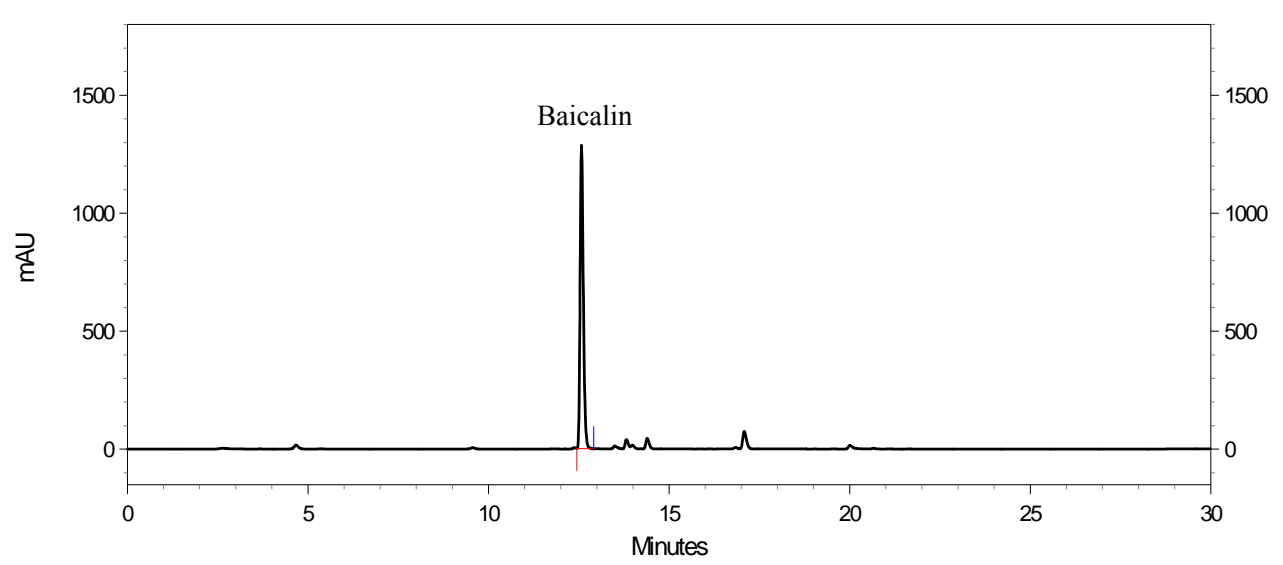

(a)

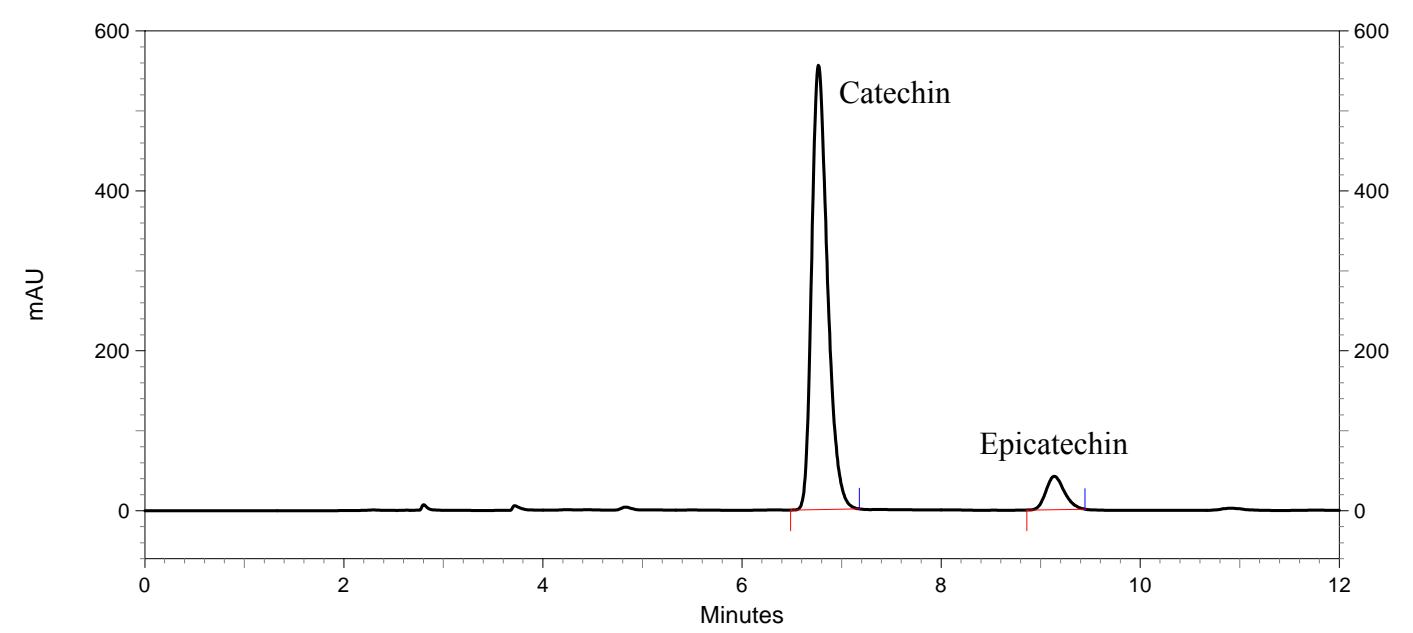

(b)

Figure 1. HPLC chromatogram of UP446. (a) Baicalin contents in UP446; (b) Catechin contents in UP446.

(OECD) Guideline, ICH Harmonised Tripartite Guideline and Korea Food and Drug Administration (KFDA) Guideline. 40 male and 40 female rats were divided into four groups of 10 animals each; 10 additional animals of each sex were used as recovery groups: five for the control and the other five for the high-dose group. UP446 $(500,1000$, or $2000 \mathrm{mg} / \mathrm{kg})$ or control group was orally administered once daily using an oral Zonde needle for 6 months. Mortality, clinical signs, body weight, food consumption and gross findings were monitored during experimental period. Ophthalmoscopic examinations were conducted on both eyes of 5 animals/sex/group in the main group and those of all animals in the recovery group at 26 weeks and 30 weeks. At the end of the study, all surviving animals were fasted overnight. 3 hours and 24 hours urine samples were collected from 5 animals/ sex/group in the main group and all animals in the recovery group prior to blood collection. Animals were anesthetized with isoflurane and blood samples were collected from the abdominal aorta and used to measure hematological and biochemical parameters. The hemato- logical parameters assessed included erythrocyte count (RBC), hemoglobin (HGB), Hematocrit (HCT), Mean corpuscular volume (MCV), Mean corpuscular hemoglobin $(\mathrm{MCH})$, Mean corpuscular hemoglobin concentration (MCHC), platelets (PLT), leukocyte count (WBC), WBC differential counting, Reticulocyte (Reti), prothrombin time (PT) and activated partial thromboplastin time (APTT). The clinical chemistry parameters assessed included alanine aminotransferase (ALT), aspartate aminotransferase (AST), alkaline phosphatase (ALP), gamma glutamyl transpeptidase (GGT), blood urea nitrogen (BUN), creatinine (Crea), total bilirubin (T-Bili), total protein (TP), albumin (Alb), Total cholesterol (T-Chol), triglycerides (TG) and glucose (Glu). After blood collection, all animals were immediately sacrificed for gross pathological examination of the internal organs. The organs such as brain, pituitary, thymus, heart, lung, liver, spleen, kidney, adrenal and sex organs were removed, blotted free of blood and weighed immediately. Histopathological examinations of animals were performed in the control group and the highest dose group, and in the 
lower dose groups when appropriate. All organs were fixed in $10 \%$ neutral-buffered formalin, and the tissue sections were prepared with hematoxylin and eosin staining before microscopic examination for pathological changes.

\subsection{Statistical Analysis}

Statistical analysis was performed using SAS program (version 9.2, SAS Institute Inc., USA). Body weights in acute oral toxicity study were analyzed utilizing Folded$\mathrm{F}$ test for homogeneity of variance. And then Student $t$ test was employed on homogeneous data for confirming significance. Body weight, food consumption, urine volume, hematology, clinical chemistry and organ weight data in 26-week repeated oral dose toxicity study were analyzed utilizing Bartlett's test for homogeneity of variance. One-way analysis of variance (ANOVA) was employed on homogeneous data; then, if significant, Dunnett's test was applied for multiple comparisons. Kruskal-Wallis test was employed on heterogeneous data. The $p$ values less than 0.05 were considered significant.

\section{Results}

\subsection{Acute Oral Toxicity Study}

To evaluate the acute oral toxicity of UP446, both sexes of rats were orally administrated UP446 at the dose of $5000 \mathrm{mg} / \mathrm{kg}$ body weight. The UP446 at a dose of 5000 $\mathrm{mg} / \mathrm{kg}$ produced no treatment related toxicity or mortality in any of the animals during 14 days of the study. In addition, no body weight loss was detected (Table 1) and all internal organs examined at necropsy were free from any gross pathological changes. Therefore, the results suggest that the lethal dose of UP446 is greater than 5000 $\mathrm{mg} / \mathrm{kg}$ in male and female rats.

\subsection{6-Week Repeated Oral Dose Toxicity Study}

No deaths and no treatment-related signs of toxicity were observed throughout 26 and 30 weeks of the study in any of the groups except one male and female in the control group. Appearance and behavior of the animals were similar for all groups of animals.

There was no statistically significant difference in body weight between the control and all treatment groups (Figure 2). Blackish stool was observed in males and females in the 500, 1000 and $2000 \mathrm{mg} / \mathrm{kg}$ UP446 groups. Soft stool was observed in one male in the $1000 \mathrm{mg} / \mathrm{kg}$ group and in some males and females in the $2000 \mathrm{mg} / \mathrm{kg}$ group (Table 2). However, these were not considered to be toxic effects because neither histopathological change in gastrointestinal tracks (GIT) nor body weight change was detected. There were no associations between treatment and the findings recorded in the ophtalmological examinations conducted at the end of the treatment and recovery periods (Tables 3 and 4). Urinalysis results also showed that no test substance related changes in males and females in the dosing groups in the main group and in the recovery group (data not shown). Some hematological parameters of female rats treated with UP446 at the dose of $2000 \mathrm{mg} / \mathrm{kg} /$ day showed statistically significant differences when compared to those of the control group. After 26-week of treatment, female rats had a slightly decreased RBC, HGB and HCT at $2000 \mathrm{mg} / \mathrm{kg}$. However, these effects were not observed in recovery group and male group (Tables $\mathbf{5}$ and $\mathbf{6}$ ). There were no test substance related changes in males and females in the dosing groups in the main group and in the 2000 $\mathrm{mg} / \mathrm{kg}$ group in the recovery group.

Serum clinical chemistry data were shown in Tables 7 and 8. In male, a statistically significant decreased in glucose at $2000 \mathrm{mg} / \mathrm{kg}$ when compared with that of the control group. There were no significant differences between treatment and control groups in females.

The absolute and relative internal organ weights of male and female rats treated with UP446 are summarized in Tables 9 and 10. In absolute and relative organ weights, no statistically significant changes were shown in the treatment group when compared to control group. However, the absolute adrenal gland weight was signifi-

Table 1. Body weight and body weight gain on day 14 of rats in the acute oral toxicity study.

\begin{tabular}{|c|c|c|c|c|c|c|}
\hline \multirow{2}{*}{ Group } & \multicolumn{5}{|c|}{ Body weight (g) } & \multirow{2}{*}{$\begin{array}{c}\text { Gain }(\mathrm{g}) \\
\text { Day 0-14 }\end{array}$} \\
\hline & Day 0 & Day 1 & Day 3 & Day 7 & Day 14 & \\
\hline \multicolumn{7}{|l|}{ Male } \\
\hline Control & $162.9 \pm 2.9$ & $189.0 \pm 4.3$ & $212.6 \pm 3.4$ & $250.5 \pm 6.6$ & $314.0 \pm 10.8$ & $151.1 \pm 8.7$ \\
\hline $\mathrm{UP} 446,5 \mathrm{~g} / \mathrm{kg}$ & $163.1 \pm 4.9$ & $188.6 \pm 4.8$ & $211.2 \pm 6.1$ & $251.6 \pm 8.7$ & $317.4 \pm 16.7$ & $154.4 \pm 13.9$ \\
\hline \multicolumn{7}{|l|}{ Feamle } \\
\hline Control & $130.9 \pm 2.9$ & $147.6 \pm 4.7$ & $162.0 \pm 4.7$ & $177.7 \pm 6.0$ & $207.3 \pm 14.1$ & $76.4 \pm 11.0$ \\
\hline $\mathrm{UP} 446,5 \mathrm{~g} / \mathrm{kg}$ & $131.1 \pm 2.9$ & $145.7 \pm 1.7$ & $163.5 \pm 6.4$ & $180.3 \pm 8.9$ & $203.0 \pm 18.1$ & $71.9 \pm 17.7$ \\
\hline
\end{tabular}

Values are means $\pm \mathrm{SD}(n=5)$. 
Table 2. Clinical signs of UP446 for 26-week repeated oral dose toxicity in male and female.

\begin{tabular}{|c|c|c|c|}
\hline & $\begin{array}{l}\text { No. of } \\
\text { animals }\end{array}$ & Clinical signs & $\begin{array}{l}\text { No. of } \\
\text { animals } \\
\text { affected }\end{array}$ \\
\hline \multicolumn{4}{|l|}{ Male } \\
\hline \multirow[t]{4}{*}{ Control } & & Decrease in food intake & 1 \\
\hline & & Decrease of fecal volume & 1 \\
\hline & & Prone position & 1 \\
\hline & & Death & 1 \\
\hline $\mathrm{UP} 446,500 \mathrm{mg} / \mathrm{kg}$ & 10 & Blackish stool & 10 \\
\hline \multirow[t]{3}{*}{ UP446, $1000 \mathrm{mg} / \mathrm{kg}$} & 10 & Blackish stool & 10 \\
\hline & & Soft stool & 1 \\
\hline & & Loss of teeth (upper teeth) & 1 \\
\hline \multirow[t]{2}{*}{$\mathrm{UP} 446,2000 \mathrm{mg} / \mathrm{kg}$} & 15 & Blackish stool & 15 \\
\hline & & Soft stool & 3 \\
\hline \multicolumn{4}{|l|}{ Female } \\
\hline \multirow[t]{2}{*}{ Control } & 15 & Prone position & 1 \\
\hline & & Death & 1 \\
\hline \multirow[t]{3}{*}{ UP446, $500 \mathrm{mg} / \mathrm{kg}$} & 10 & Blackish stool & 15 \\
\hline & & Wound (anterior neck) & 1 \\
\hline & & $\begin{array}{l}\text { Crust formation } \\
\text { (anterior neck) }\end{array}$ & 1 \\
\hline UP446, $1000 \mathrm{mg} / \mathrm{kg}$ & 10 & Blackish stool & 10 \\
\hline \multirow[t]{2}{*}{$\mathrm{UP} 446,2000 \mathrm{mg} / \mathrm{kg}$} & 15 & Blackish stool & 15 \\
\hline & & Soft stool & 4 \\
\hline
\end{tabular}

cantly increased in female recovery group only. That organ did not show any histopathological changes and just exhibit in female recovery group only. There were no test substance-related changes in male and females in the dosing groups in the main group and in the $2000 \mathrm{mg} / \mathrm{kg}$ group in the recovery group.

The gross and histopathological examination revealed no findings related to the treatment (data not shown). The findings were observed in a few animals or were equally distributed among the groups, including in the control. They were considered spontaneous changes that occur commonly in normal rats of this strain and age, unrelated to the administration of UP446.

\section{Discussion}

The present study demonstrated the comprehensive safety profile of standardized composition of bioflavonoids extracted from $S$. baicalensis and A. catechu at a dose as high as $5000 \mathrm{mg} / \mathrm{kg}$ in the acute toxicity study and $2000 \mathrm{mg} / \mathrm{kg}$ in the 26 -week repeated oral administration of. Previous study confirmed the safety of UP446

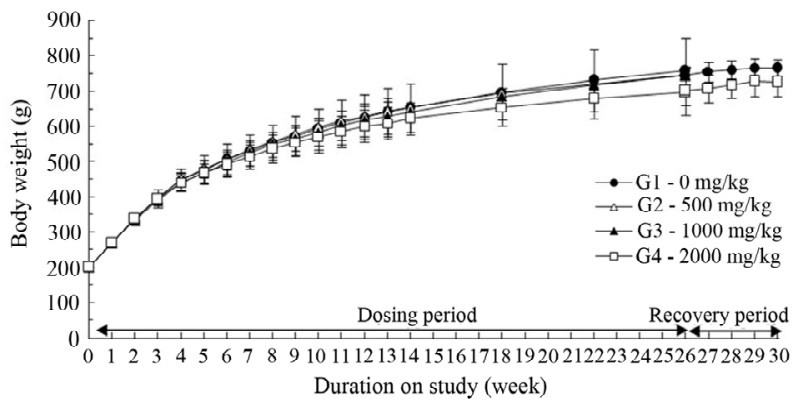

(a)

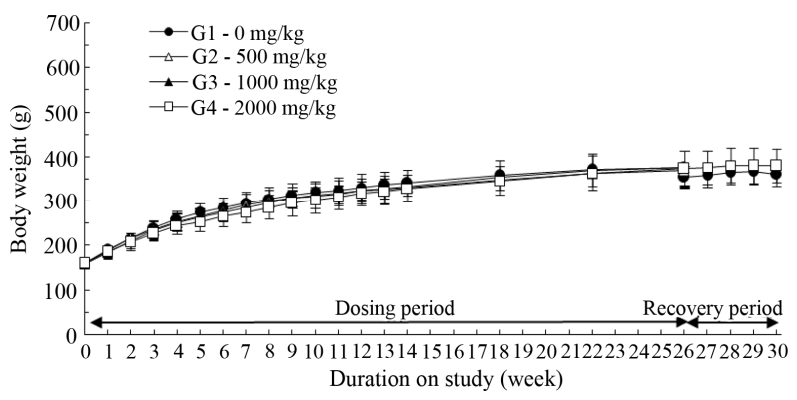

(b)

Figure 2. Effects of oral administration of UP446 for 26week repeated oral dose toxicity with 4-week recovery period on mean body weights. (a) Body weight of male group (G1: control; G2: UP446 500 mg/kg; G3: UP446 1000 mg/kg; G4: $2000 \mathrm{mg} / \mathrm{kg}$ ); (b) Body weight of female group (G1: control; G2: UP446 500 mg/kg; G3: UP446 1000 mg/kg; G4: $2000 \mathrm{mg} / \mathrm{kg})$. Data expressed as mean \pm SD.

in 3 months repeated toxicity study using rats. NOAEL was assumed to be over $1000 \mathrm{mg} / \mathrm{kg} /$ day [23]. However, the systemic toxic effects of acute and long term treatment should be evaluated since the target diseases of UP446 are chronic arthritis that required long term treatment.

In the acute toxicity study, UP446 caused neither treatment related signs of toxicity nor mortality during 14 days of the study. No test substance related effects on body weight and necropsy were observed in any animals at $5000 \mathrm{mg} / \mathrm{kg}$ group. Therefore, the lethal dose of UP446 is greater than $5000 \mathrm{mg} / \mathrm{kg}$ in male and female rats.

In 26-week repeated oral dose toxicity study, no deaths and no treatment related signs were observed in animals of all groups except one male and female of control group. Food consumption of UP446 treated groups were found to be insignificant in male when compared to the control groups but significantly increased food consumption was observed in females in the $2000 \mathrm{mg} / \mathrm{kg}$ group at week 14 when compared to the control group (data not shown). However, these changes in food consumption were not considered to be test substance related effects since these were observed incidentally. Blackish stool and soft stool was observed in one male in the 1000 
of Scutellaria Extract and Acacia Extract in Rats

Table 3. Urinalysis results of UP446 for 26-week repeated oral dose toxicity with 4-week recovery period in male rats.

\begin{tabular}{|c|c|c|c|c|c|c|c|}
\hline \multirow{2}{*}{$\begin{array}{c}\text { Parameters } \\
\text { Group/dose (mg/kg) }\end{array}$} & \multirow[t]{2}{*}{ Grade } & \multicolumn{4}{|c|}{ Main group (mg/kg) } & \multicolumn{2}{|c|}{ Recovery group (mg/kg) } \\
\hline & & Control & UP446, 500 & UP446, 1000 & UP446, 2000 & Control & UP446, 2000 \\
\hline No. of animals & & 5 & 5 & 5 & 5 & 5 & 5 \\
\hline Volume (mL) & & $14.4 \pm 6.7$ & $17.0 \pm 3.9$ & $18.8 \pm 8.3$ & $12.2 \pm 3.0$ & $9.4 \pm 2.4$ & $8.3 \pm 2.9$ \\
\hline \multirow[t]{2}{*}{ Color } & Pale yellow & 4 & & & & & \\
\hline & Yellow & 1 & 5 & 5 & 5 & 5 & 5 \\
\hline \multirow[t]{3}{*}{ Transparency } & Clear & 5 & 5 & 5 & 5 & 5 & 5 \\
\hline & Mild turbidity & & & & & & \\
\hline & Turbidity & & & & & & \\
\hline \multirow[t]{6}{*}{$\mathrm{pH}$} & 5 & & & & & & \\
\hline & 6 & & & & & & \\
\hline & 6.5 & & 1 & & 2 & & \\
\hline & 7 & 2 & 3 & 2 & 3 & & 2 \\
\hline & 8 & 2 & 1 & 3 & & 4 & \\
\hline & 9 & 1 & & & & 1 & 3 \\
\hline \multirow[t]{5}{*}{ Protein $(\mathrm{mg} / \mathrm{dL})$} & & 3 & 5 & 2 & 4 & & 1 \\
\hline & 25 & 2 & & 2 & 1 & 4 & 3 \\
\hline & 75 & & & 1 & & & 1 \\
\hline & 150 & & & & & 1 & \\
\hline & 500 & & & & & & \\
\hline \multirow[t]{5}{*}{ Glucose (mg/dL) } & Normal & 5 & 5 & 5 & 5 & 5 & 5 \\
\hline & 50 & & & & & & \\
\hline & 100 & & & & & & \\
\hline & 300 & & & & & & \\
\hline & 1000 & & & & & & \\
\hline \multirow[t]{5}{*}{ Ketone body (mg/dL) } & & 5 & 5 & 5 & 5 & 5 & 5 \\
\hline & 5 & & & & & & \\
\hline & 15 & & & & & & \\
\hline & 50 & & & & & & \\
\hline & 100 & & & & & & \\
\hline \multirow[t]{4}{*}{ Bilirubin (mg/dL) } & & 5 & 5 & 5 & 5 & 5 & 5 \\
\hline & 1 & & & & & & \\
\hline & 3 & & & & & & \\
\hline & 4 & & & & & & \\
\hline \multirow[t]{5}{*}{ Occult blood $($ Ery $/ \mu \mathrm{L})$} & & 5 & 5 & 5 & 5 & 4 & 5 \\
\hline & 10 & & & & & 1 & \\
\hline & 25 & & & & & & \\
\hline & 50 & & & & & & \\
\hline & 150 & & & & & & \\
\hline
\end{tabular}




\section{Continued}

\begin{tabular}{|c|c|c|c|c|c|c|c|}
\hline & 250 & & & & & & \\
\hline \multirow[t]{4}{*}{ Cast $^{\ddagger}$} & 0 & 5 & 5 & 5 & 5 & 5 & 5 \\
\hline & $1-5$ & & & & & & \\
\hline & $6-10$ & & & & & & \\
\hline & $>10$ & & & & & & \\
\hline \multirow[t]{4}{*}{ Epithelial cell ${ }^{\ddagger}$} & 0 & 5 & 5 & 5 & 5 & 5 & 5 \\
\hline & $1-5$ & & & & & & \\
\hline & $6-10$ & & & & & & \\
\hline & $>10$ & & & & & & \\
\hline \multirow[t]{5}{*}{ Leukocyte $^{\ddagger}$} & 0 & 5 & 5 & 5 & 5 & 5 & 5 \\
\hline & $1-10$ & & & & & & \\
\hline & $11-50$ & & & & & & \\
\hline & $51-100$ & & & & & & \\
\hline & $>100$ & & & & & & \\
\hline \multirow[t]{5}{*}{ Erythrocyte } & 0 & 5 & 5 & 5 & 5 & 5 & 5 \\
\hline & $1-10$ & & & & & & \\
\hline & $11-50$ & & & & & & \\
\hline & $51-100$ & & & & & & \\
\hline & $>100$ & & & & & & \\
\hline \multirow[t]{7}{*}{ Specific gravity } & $1.000-1.010$ & & & & & & \\
\hline & $1.011-1.020$ & & & & & & \\
\hline & $1.021-1.030$ & & & 1 & & & \\
\hline & $1.031-1.040$ & 2 & 3 & 1 & & & \\
\hline & $1.041-1.050$ & 1 & 2 & 2 & 3 & & \\
\hline & $1.051-1.060$ & 2 & & 1 & 1 & 1 & 1 \\
\hline & $>1.060$ & & & & 1 & 4 & 4 \\
\hline
\end{tabular}

Sediment.

$\mathrm{mg} / \mathrm{kg}$ group and in some males and females in the 2000 $\mathrm{mg} / \mathrm{kg}$ group. Although those symptoms were considered to be test substance related effects, these were not toxic effects since there were no changes on gastrointestinal track at necropsy and histopathology, and there were no effects on the body weights.

Blood is an important index of physiological and pathological status in man and animal [25]. Some statistically significant differences were observed from the results of hematological parameters of female rats treated with UP446 at the dose of $2000 \mathrm{mg} / \mathrm{kg} /$ day. However, all differences in hematology parameters were not considered to be test substance-related effects because of the small magnitude of the difference with in the normal ranges, the lack of a clear dose response and the lack of relationship without histological correlation. There are strong evidences to suggest that both the traditional non selective non-steroidal anti-inflammatory drugs (NSAIDs) and the selective cyclooxygenase-2 (COX-2) inhibitors are associated with an increased risk of thrombotic events and adverse gastrointestinal effects [26,27]. To confirm the impact of UP466 on blood coagulation, we measured pro-thrombin and activated partial thromboplastin times after 26-week of repeated oral dose of rats at dose rate as high as $2000 \mathrm{mg} / \mathrm{kg} / \mathrm{day}$, an equivalent of $22.68 \mathrm{~g}$ average human daily dose. There was no 

of Scutellaria Extract and Acacia Extract in Rats

Table 4. Urinalysis results of UP446 for 26-week repeated oral dose toxicity with 4-week recovery period in female rats.

\begin{tabular}{|c|c|c|c|c|c|c|c|}
\hline \multirow{2}{*}{$\begin{array}{c}\text { Parameters } \\
\text { Group/dose (mg/kg) }\end{array}$} & \multirow[t]{2}{*}{ Grade } & \multicolumn{4}{|c|}{ Main group (mg/kg) } & \multicolumn{2}{|c|}{ Recovery group (mg/kg) } \\
\hline & & Control & UP446, 500 & UP446, 1000 & UP446, 2000 & Control & UP446, 2000 \\
\hline No. of animals & & 5 & 5 & 5 & 5 & 5 & 5 \\
\hline Volume (mL) & & $8.2 \pm 2.5$ & $6.6 \pm 2.7$ & $6.6 \pm 3.5$ & $9.0 \pm 2.8$ & $6.9 \pm 2.6$ & $9.1 \pm 4.5$ \\
\hline \multirow[t]{2}{*}{ Color } & Pale yellow & 5 & & & & & \\
\hline & Yellow & & 5 & 5 & 5 & 5 & 5 \\
\hline \multirow[t]{3}{*}{ Transparency } & Clear & 5 & 5 & 5 & 5 & 5 & 4 \\
\hline & Mild turbidity & & & & & & \\
\hline & Turbidity & & & & & & 1 \\
\hline \multirow[t]{6}{*}{$\mathrm{pH}$} & 5 & & & & & & \\
\hline & 6 & & & & 1 & 1 & \\
\hline & 6.5 & & & 1 & & & \\
\hline & 7 & 1 & & & 3 & 2 & 2 \\
\hline & 8 & 1 & 4 & 1 & & 1 & 1 \\
\hline & 9 & 3 & 1 & 3 & 1 & 1 & 2 \\
\hline \multirow[t]{5}{*}{ Protein $(\mathrm{mg} / \mathrm{dL})$} & & 5 & 2 & 5 & 5 & 2 & 1 \\
\hline & 25 & & 3 & & & 3 & 2 \\
\hline & 75 & & & & & & 2 \\
\hline & 150 & & & & & & \\
\hline & 500 & & & & & & \\
\hline \multirow[t]{5}{*}{ Glucose (mg/dL) } & Normal & 5 & 5 & 5 & 5 & 5 & 5 \\
\hline & 50 & & & & & & \\
\hline & 100 & & & & & & \\
\hline & 300 & & & & & & \\
\hline & 1000 & & & & & & \\
\hline \multirow[t]{5}{*}{ Ketone body (mg/dL) } & & 5 & 5 & 5 & 5 & 5 & 5 \\
\hline & 5 & & & & & & \\
\hline & 15 & & & & & & \\
\hline & 50 & & & & & & \\
\hline & 100 & & & & & & \\
\hline \multirow[t]{4}{*}{ Bilirubin $(\mathrm{mg} / \mathrm{dL})$} & & 5 & 5 & 5 & 5 & 5 & 5 \\
\hline & 1 & & & & & & \\
\hline & 3 & & & & & & \\
\hline & 4 & & & & & & \\
\hline \multirow[t]{6}{*}{ Occult blood $($ Ery $/ \mu \mathrm{L})$} & & 5 & 5 & 5 & 5 & 5 & 5 \\
\hline & 10 & & & & & & \\
\hline & 25 & & & & & & \\
\hline & 50 & & & & & & \\
\hline & 150 & & & & & & \\
\hline & 250 & & & & & & \\
\hline \multirow[t]{4}{*}{ Cast $^{+}$} & 0 & 5 & 5 & 5 & 5 & 5 & 5 \\
\hline & $1-5$ & & & & & & \\
\hline & $6-10$ & & & & & & \\
\hline & $>10$ & & & & & & \\
\hline
\end{tabular}




\section{Continued}

\begin{tabular}{|c|c|c|c|c|c|c|c|}
\hline \multirow[t]{4}{*}{ Epithelial cell $^{\ddagger}$} & 0 & 5 & 5 & 5 & 5 & 5 & 5 \\
\hline & $1-5$ & & & & & & \\
\hline & $6-10$ & & & & & & \\
\hline & $>10$ & & & & & & \\
\hline \multirow[t]{5}{*}{ Leukocyte ${ }^{\ddagger}$} & 0 & 5 & 5 & 5 & 5 & 5 & 5 \\
\hline & $1-10$ & & & & & & \\
\hline & $11-50$ & & & & & & \\
\hline & $51-100$ & & & & & & \\
\hline & $>100$ & & & & & & \\
\hline \multirow[t]{5}{*}{ Erythrocyte ${ }^{\ddagger}$} & 0 & 5 & 5 & 5 & 5 & 5 & 5 \\
\hline & $1-10$ & & & & & & \\
\hline & $11-50$ & & & & & & \\
\hline & $51-100$ & & & & & & \\
\hline & $>100$ & & & & & & \\
\hline \multirow[t]{7}{*}{ Specific gravity } & $1.000-1.010$ & & & & & & \\
\hline & $1.011-1.020$ & & & & & & \\
\hline & $1.021-1.030$ & & 1 & & & 1 & 1 \\
\hline & $1.031-1.040$ & 4 & 2 & 2 & 2 & 1 & 1 \\
\hline & $1.041-1.050$ & 1 & & 1 & 3 & 2 & 1 \\
\hline & $1.051-1.060$ & & 1 & 1 & & 1 & 2 \\
\hline & $>1.060$ & & 1 & 1 & & & \\
\hline
\end{tabular}

${ }^{\dagger}$ Sediment.

Table 5. Mean hematological parameters of UP446 for 26-week repeated oral dose toxicity with 4-week recovery period in male rats.

\begin{tabular}{|c|c|c|c|c|c|c|}
\hline \multirow{2}{*}{ Parameters } & \multicolumn{4}{|c|}{ Main group (mg/kg) } & \multicolumn{2}{|c|}{ Recovery group (mg/kg) } \\
\hline & Control & UP446, 500 & UP446, 1000 & UP446, 2000 & Control & UP446, 2000 \\
\hline $\mathrm{RBC}\left(\times 10^{6}\right.$ cells $\left./ \mu \mathrm{L}\right)$ & $8.74 \pm 0.26$ & $8.55 \pm 0.23$ & $8.64 \pm 0.35$ & $8.61 \pm 0.38$ & $8.57 \pm 0.16$ & $8.69 \pm 0.34$ \\
\hline $\mathrm{HGB}(\mathrm{g} / \mathrm{dL})$ & $14.8 \pm 0.6$ & $14.6 \pm 0.6$ & $14.8 \pm 0.7$ & $14.9 \pm 0.5$ & $14.6 \pm 0.5$ & $15.2 \pm 0.5$ \\
\hline HCT (\%) & $45.8 \pm 2.0$ & $44.7 \pm 1.7$ & $45.4 \pm 2.0$ & $45.1 \pm 1.4$ & $44.5 \pm 1.3$ & $45.6 \pm 1.6$ \\
\hline MCV (fL) & $52.4 \pm 1.9$ & $52.3 \pm 2.1$ & $52.6 \pm 1.5$ & $52.4 \pm 1.5$ & $52.0 \pm 2.2$ & $52.5 \pm 1.0$ \\
\hline $\mathrm{MCH}(\mathrm{pg})$ & $17.0 \pm 0.5$ & $17.1 \pm 0.7$ & $17.2 \pm 0.5$ & $17.3 \pm 0.5$ & $17.1 \pm 0.8$ & $17.6 \pm 0.3$ \\
\hline $\mathrm{MCHC}(\mathrm{g} / \mathrm{dL})$ & $32.4 \pm 0.5$ & $32.8 \pm 0.5$ & $32.7 \pm 0.6$ & $33.0 \pm 0.4$ & $32.9 \pm 0.3$ & $33.4 \pm 0.2^{*}$ \\
\hline $\operatorname{PLT}\left(\times 10^{3}\right.$ cells $\left./ \mu \mathrm{L}\right)$ & $1068 \pm 93$ & $1083 \pm 87$ & $1092 \pm 156$ & $1135 \pm 98$ & $1158 \pm 138$ & $1051 \pm 92$ \\
\hline Reti (\%) & $2.2 \pm 0.3$ & $2.3 \pm 0.5$ & $2.2 \pm 0.8$ & $2.0 \pm 0.4$ & $2.5 \pm 0.3$ & $2.3 \pm 0.4$ \\
\hline $\mathrm{WBC}\left(\times 10^{3}\right.$ cells $\left./ \mu \mathrm{L}\right)$ & $7.13 \pm 0.81$ & $6.96 \pm 2.16$ & $6.53 \pm 1.47$ & $6.58 \pm 1.87$ & $6.98 \pm 2.47$ & $6.67 \pm 1.61$ \\
\hline NEU & $20.1 \pm 3.7$ & $20.7 \pm 9.0$ & $17.8 \pm 4.9$ & $24.6 \pm 6.1$ & $26.3 \pm 6.1$ & $14.9 \pm 2.0^{* *}$ \\
\hline LYM & $72.5 \pm 4.5$ & $72.2 \pm 9.8$ & $76.2 \pm 5.2$ & $69.1 \pm 6.2$ & $67.0 \pm 5.8$ & $78.1 \pm 1.9^{* *}$ \\
\hline MONO & $3.7 \pm 1.0$ & $3.8 \pm 1.6$ & $2.8 \pm 1.2$ & $3.0 \pm 0.8$ & $4.0 \pm 0.9$ & $4.1 \pm 1.1$ \\
\hline EOS & $1.3 \pm 0.4$ & $1.3 \pm 0.4$ & $1.1 \pm 0.2$ & $1.1 \pm 0.3$ & $1.4 \pm 0.6$ & $1.6 \pm 0.8$ \\
\hline BASO & $0.3 \pm 0.1$ & $0.3 \pm 0.1$ & $0.3 \pm 0.1$ & $0.3 \pm 0.1$ & $0.3 \pm 0.1$ & $0.2 \pm 0.1$ \\
\hline PT (sec) & $15.9 \pm 0.6$ & $16.1 \pm 0.6$ & $15.6 \pm 1.1$ & $15.8 \pm 0.7$ & $16.3 \pm 0.4$ & $16.0 \pm 1.0$ \\
\hline APTT (sec) & $16.6 \pm 0.9$ & $16.5 \pm 1.2$ & $16.4 \pm 1.7$ & $16.3 \pm 1.2$ & $17.1 \pm 1.4$ & $17.4 \pm 0.9$ \\
\hline
\end{tabular}

Mean hematological parameters were calculated and data were expressed as mean $\pm \operatorname{SD}(n=10$, except control group; $n=9$, Recovery group; $n=5)$. Significantly different from control by Student t-test for recovery ${ }^{*} p<0.05,{ }^{* *} p<0.01$. 
Table 6. Mean hematological parameters of UP446 for 26-week repeated oral dose toxicity with 4-week recovery period in female rats.

\begin{tabular}{|c|c|c|c|c|c|c|}
\hline \multirow{2}{*}{ Parameters } & \multicolumn{4}{|c|}{ Main group (mg/kg) } & \multicolumn{2}{|c|}{ Recovery group (mg/kg) } \\
\hline & Control & UP446, 500 & UP446, 1000 & UP446, 2000 & Control & UP446, 2000 \\
\hline $\mathrm{RBC}\left(\times 10^{6}\right.$ cells $\left./ \mu \mathrm{L}\right)$ & $7.78 \pm 0.28$ & $7.82 \pm 0.30$ & $7.71 \pm 7.44$ & $7.44 \pm 0.22^{*}$ & $7.83 \pm 0.50$ & $7.74 \pm 0.11$ \\
\hline HGB $(\mathrm{g} / \mathrm{dL})$ & $14.5 \pm 0.5$ & $14.1 \pm 0.4$ & $14.5 \pm 0.4$ & $13.7 \pm 0.3^{* *}$ & $14.8 \pm 0.2$ & $14.4 \pm 0.5$ \\
\hline HCT (\%) & $43.2 \pm 1.8$ & $42.3 \pm 1.2$ & $43.4 \pm 1.4$ & $41.2 \pm 1.0^{* *}$ & $43.0 \pm 1.0$ & $42.1 \pm 1.6$ \\
\hline MCV (fL) & $55.6 \pm 1.6$ & $54.1 \pm 1.2^{*}$ & $56.3 \pm 1.3$ & $55.4 \pm 1.0$ & $55.1 \pm 3.0$ & $54.4 \pm 1.4$ \\
\hline $\mathrm{MCH}(\mathrm{pg})$ & $18.6 \pm 0.3$ & $18.1 \pm 0.5^{*}$ & $18.8 \pm 0.4$ & $18.4 \pm 0.3$ & $19.0 \pm 1.1$ & $18.7 \pm 0.5$ \\
\hline $\mathrm{MCHC}(\mathrm{g} / \mathrm{dL})$ & $33.4 \pm 0.5$ & $33.4 \pm 0.3$ & $33.3 \pm 0.4$ & $33.3 \pm 0.4$ & $34.6 \pm 0.9$ & $34.3 \pm 0.6$ \\
\hline $\operatorname{PLT}\left(\times 10^{3}\right.$ cells $\left./ \mu \mathrm{L}\right)$ & $1029 \pm 130$ & $1149 \pm 200$ & $1049 \pm 138$ & $1011 \pm 62$ & $1053 \pm 101$ & $1022 \pm 86$ \\
\hline Reti (\%) & $2.0 \pm 0.4$ & $1.9 \pm 0.2$ & $2.1 \pm 0.3$ & $1.8 \pm 0.4$ & $2.2 \pm 0.8$ & $1.8 \pm 0.4$ \\
\hline $\mathrm{WBC}\left(\times 10^{3}\right.$ cells $\left./ \mu \mathrm{L}\right)$ & $3.13 \pm 0.96$ & $3.74 \pm 1.13$ & $3.32 \pm 0.71$ & $3.59 \pm 1.04$ & $4.82 \pm 1.85$ & $3.58 \pm 1.01$ \\
\hline NEU & $19.5 \pm 6.0$ & $19.8 \pm 6.8$ & $20.5 \pm 4.1$ & $16.8 \pm 3.7$ & $16.1 \pm 8.4$ & $14.2 \pm 3.3$ \\
\hline LYM & $73.6 \pm 7.6$ & $73.5 \pm 7.1$ & $72.4 \pm 4.5$ & $76.4 \pm 5.3$ & $76.3 \pm 11.8$ & $78.9 \pm 2.8$ \\
\hline MONO & $3.6 \pm 1.3$ & $3.3 \pm 1.1$ & $3.6 \pm 1.1$ & $3.4 \pm 1.7$ & $3.7 \pm 2.3$ & $3.4 \pm 0.8$ \\
\hline EOS & $1.5 \pm 0.9$ & $1.6 \pm 0.5$ & $1.5 \pm 0.4$ & $1.5 \pm 0.5$ & $1.4 \pm 0.6$ & $1.5 \pm 0.5$ \\
\hline BASO & $0.3 \pm 0.1$ & $0.3 \pm 0.1$ & $0.3 \pm 0.1$ & $0.3 \pm 0.1$ & $0.2 \pm 0.1$ & $0.3 \pm 0.2$ \\
\hline $\mathrm{PT}(\mathrm{sec})$ & $15.1 \pm 1.0$ & $15.3 \pm 1.0$ & $15.2 \pm 0.7$ & $15.5 \pm 0.6$ & $15.3 \pm 0.7$ & $15.2 \pm 0.3$ \\
\hline APTT (sec) & $17.1 \pm 0.8$ & $16.8 \pm 1.4$ & $16.6 \pm 0.5$ & $17.0 \pm 1.0$ & $16.0 \pm 1.7$ & $16.4 \pm 0.5$ \\
\hline
\end{tabular}

Mean hematological parameters were calculated and data were expressed as mean $\pm \mathrm{SD}(n=10$, except control group; $n=9)$. Significantly different from control by Dunnett's $t$-test: ${ }^{*} p<0.05,{ }^{* *} p<0.01$.

Table 7. Mean clinical chemistry parameters of UP446 for 26-week repeated oral dose toxicity with 4-week recovery period in male rats.

\begin{tabular}{ccccccc}
\hline \multirow{2}{*}{ Parameters } & \multicolumn{3}{c}{ Main group $(\mathrm{mg} / \mathrm{kg})$} & \multicolumn{2}{c}{ Recovery group $(\mathrm{mg} / \mathrm{kg})$} \\
\cline { 2 - 7 } & Control & UP446,500 & UP446, 1000 & UP446, 2000 & Control & UP446, 2000 \\
\hline ALT (U/L) & $43.7 \pm 16.5$ & $36.6 \pm 13.2$ & $30.9 \pm 5.7$ & $35.8 \pm 9.8$ & $33.7 \pm 6.9$ & $31.5 \pm 7.1$ \\
AST (U/L) & $90.2 \pm 24.5$ & $88.9 \pm 28.9$ & $85.1 \pm 17.5$ & $81.7 \pm 25.2$ & $94.6 \pm 26.6$ & $110.6 \pm 20.8$ \\
ALP (U/L) & $190.5 \pm 52.5$ & $223.4 \pm 37.4$ & $209.7 \pm 25.7$ & $243.0 \pm 43.8$ & $225.2 \pm 48.8$ & $200.9 \pm 27.0$ \\
GGT (U/L) & $0.23 \pm 0.14$ & $0.40 \pm 0.30$ & $0.81 \pm 0.34^{* *}$ & $0.46 \pm 0.30$ & $1.15 \pm 0.57$ & $0.88 \pm 0.39$ \\
Glu (mg/dL) & $161 \pm 15$ & $154 \pm 10$ & $151 \pm 10$ & $139 \pm 12^{* *}$ & $165 \pm 13$ & $174 \pm 19$ \\
BUN (mg/dL) & $10.9 \pm 0.8$ & $10.4 \pm 1.2$ & $10.3 \pm 1.5$ & $9.4 \pm 1.3$ & $14.0 \pm 1.5$ & $12.1 \pm 1.0^{*}$ \\
Crea (mg/dL) & $0.52 \pm 0.05$ & $0.50 \pm 0.04$ & $0.47 \pm 0.06$ & $0.48 \pm 0.05$ & $0.52 \pm 0.05$ & $0.53 \pm 0.04$ \\
T-Bili (mg/dL) & $0.06 \pm 0.03$ & $0.06 \pm 0.02$ & $0.05 \pm 0.02$ & $0.04 \pm 0.01$ & $0.07 \pm 0.02$ & $0.07 \pm 0.02$ \\
T-Chol (mg/dL) & $99 \pm 22$ & $97 \pm 24$ & $88 \pm 26$ & $95 \pm 35$ & $110 \pm 17$ & $106 \pm 10$ \\
TG (mg/dL) & $88 \pm 45$ & $105 \pm 61$ & $106 \pm 80$ & $86 \pm 44$ & $87 \pm 21$ & $73 \pm 17$ \\
TP (g/dL) & $6.1 \pm 0.3$ & $6.0 \pm 0.2$ & $6.1 \pm 0.3$ & $6.1 \pm 0.3$ & $6.4 \pm 0.3$ & $6.3 \pm 0.2$ \\
Alb (g/dL) & $2.3 \pm 0.1$ & $2.3 \pm 0.1$ & $2.3 \pm 0.1$ & $2.5 \pm 0.1$ & $2.4 \pm 0.2$ & $2.4 \pm 0.1$ \\
A/G ratio & $0.61 \pm 0.03$ & $0.63 \pm 0.04$ & $0.63 \pm 0.03$ & $0.67 \pm 0.07$ & $0.62 \pm 0.05$ & $0.62 \pm 0.04$ \\
P (mg/dL) & $5.53 \pm 0.73$ & $5.42 \pm 0.61$ & $5.75 \pm 0.62$ & $5.47 \pm 0.73$ & $5.98 \pm 0.30$ & $5.47 \pm 0.25^{*}$ \\
Ca (mg/dL) & $10.3 \pm 0.4$ & $10.1 \pm 0.4$ & $10.2 \pm 0.2$ & $10.2 \pm 0.2$ & $10.1 \pm 0.3$ & $10.0 \pm 0.3$ \\
Na (mmol/L) & $142 \pm 1$ & $142 \pm 1$ & $143 \pm 1$ & $143 \pm 1^{*}$ & $141 \pm 1$ & $141 \pm 1$ \\
K (mmol/L) & $4.7 \pm 0.2$ & $4.6 \pm 0.3$ & $4.6 \pm 0.2$ & $4.5 \pm 0.2$ & $5.0 \pm 0.3$ & $4.9 \pm 0.1$ \\
Cl (mmol/L) & $105 \pm 1$ & $105 \pm 2$ & $105 \pm 1$ & $105 \pm 1$ & $103 \pm 1$ & $104 \pm 1$ \\
\hline
\end{tabular}

Mean clinical chemistry parameters were calculated and data were expressed as mean \pm SD. Significantly different from control by Dunnett's $t$-test for main group and Student $t$-test for recovery group: ${ }^{*} p<0.05, \stackrel{* *}{p}<0.01$. 
Table 8. Mean clinical chemistry parameters of UP446 for 26-week repeated oral dose toxicity with 4 week recovery period in female rats.

\begin{tabular}{|c|c|c|c|c|c|c|}
\hline \multirow{2}{*}{ Parameters } & \multicolumn{4}{|c|}{ Main group (mg/kg) } & \multicolumn{2}{|c|}{ Recovery group (mg/kg) } \\
\hline & Control & UP446, 500 & UP446, 1000 & UP446, 2000 & Control & UP446, 2000 \\
\hline $\operatorname{ALT}(\mathrm{U} / \mathrm{L})$ & $64.1 \pm 41.3$ & $48.4 \pm 31.4$ & $68.6 \pm 83.2$ & $60.2 \pm 50.0$ & $77.1 \pm 62.3$ & $29.7 \pm 6.6$ \\
\hline AST (U/L) & $142.1 \pm 89.9$ & $105.4 \pm 41.5$ & $154.6 \pm 154.3$ & $166.5 \pm 161.7$ & $207.1 \pm 162.0$ & $98.9 \pm 31.1$ \\
\hline $\mathrm{ALP}(\mathrm{U} / \mathrm{L})$ & $92.6 \pm 16.0$ & $108.5 \pm 35.9$ & $93.6 \pm 24.4$ & $94.0 \pm 16.1$ & $81.1 \pm 12.1$ & $77.1 \pm 22.3$ \\
\hline GGT (U/L) & $0.94 \pm 0.34$ & $0.78 \pm 1.00$ & $0.67 \pm 0.45$ & $0.94 \pm 0.60$ & $0.75 \pm 0.43$ & $0.37 \pm 0.32$ \\
\hline Glu (mg/dL) & $162 \pm 18$ & $154 \pm 14$ & $156 \pm 18$ & $148 \pm 13$ & $170 \pm 29$ & $149 \pm 7$ \\
\hline BUN (mg/dL) & $14.0 \pm 1.6$ & $13.3 \pm 1.5$ & $12.8 \pm 2.7$ & $12.8 \pm 1.7$ & $14.9 \pm 1.3$ & $13.5 \pm 1.2$ \\
\hline Crea $(\mathrm{mg} / \mathrm{dL})$ & $0.55 \pm 0.05$ & $0.54 \pm 0.08$ & $0.54 \pm 0.05$ & $0.57 \pm 0.08$ & $0.57 \pm 0.09$ & $0.51 \pm 0.04$ \\
\hline T-Bili (mg/dL) & $0.09 \pm 0.02$ & $0.07 \pm 0.02$ & $0.09 \pm 0.02$ & $0.08 \pm 0.02$ & $0.13 \pm 0.03$ & $0.11 \pm 0.02$ \\
\hline T-Chol (mg/dL) & $116 \pm 28$ & $111 \pm 19$ & $113 \pm 31$ & $122 \pm 25$ & $107 \pm 25$ & $124 \pm 19$ \\
\hline $\mathrm{TG}(\mathrm{mg} / \mathrm{dL})$ & $32 \pm 10$ & $31 \pm 9$ & $35 \pm 14$ & $43 \pm 16$ & $52 \pm 19$ & $79 \pm 25$ \\
\hline $\mathrm{TP}(\mathrm{g} / \mathrm{dL})$ & $7.0 \pm 0.4$ & $7.0 \pm 0.4$ & $7.0 \pm 0.4$ & $7.0 \pm 0.3$ & $6.8 \pm 0.4$ & $7.1 \pm 0.4$ \\
\hline $\mathrm{Alb}(\mathrm{g} / \mathrm{dL})$ & $3.2 \pm 0.2$ & $3.2 \pm 0.3$ & $3.2 \pm 0.3$ & $3.4 \pm 0.2$ & $3.0 \pm 0.1$ & $3.2 \pm 0.2$ \\
\hline $\mathrm{A} / \mathrm{G}$ ratio & $0.84 \pm 0.03$ & $0.84 \pm 0.09$ & $0.84 \pm 0.06$ & $0.96 \pm 0.09$ & $0.79 \pm 0.05$ & $0.83 \pm 0.04$ \\
\hline $\mathrm{P}(\mathrm{mg} / \mathrm{dL})$ & $4.44 \pm 0.82$ & $4.35 \pm 0.58$ & $4.47 \pm 0.64$ & $4.37 \pm 1.05$ & $4.28 \pm 0.36$ & $3.82 \pm 0.53$ \\
\hline $\mathrm{Ca}(\mathrm{mg} / \mathrm{dL})$ & $10.5 \pm 0.3$ & $10.5 \pm 0.4$ & $10.6 \pm 0.4$ & $10.7 \pm 0.3$ & $10.1 \pm 0.3$ & $10.2 \pm 0.2$ \\
\hline $\mathrm{Na}(\mathrm{mmol} / \mathrm{L})$ & $142 \pm 1$ & $142 \pm 1$ & $143 \pm 1$ & $143 \pm 1$ & $141 \pm 1$ & $142 \pm 1$ \\
\hline $\mathrm{K}(\mathrm{mmol} / \mathrm{L})$ & $4.0 \pm 0.3$ & $4.0 \pm 0.2$ & $4.1 \pm 0.3$ & $4.1 \pm 0.3$ & $3.9 \pm 0.2$ & $4.0 \pm 0.3$ \\
\hline $\mathrm{Cl}(\mathrm{mmol} / \mathrm{L})$ & $105 \pm 2$ & $105 \pm 3$ & $105 \pm 2$ & $104 \pm 1$ & $104 \pm 1$ & $104 \pm 1$ \\
\hline
\end{tabular}

Mean clinical chemistry parameters were calculated and data were expressed as mean $\pm \mathrm{SD}$.

treatment related or statistically significant changes in coagulation parameters in each treatment group for both males and females, when compared to the control group. Adverse effects of traditional NSAIDs causing gastrointestinal lesions dosed with as little ibuprofen as $2 \mathrm{mg} / \mathrm{kg} /$ day, equivalent to approximately $140 \mathrm{mg}$ /day in humans has been demonstrated in rats $[23,28]$. The current study substantiate this findings in that after 26-week of repeated oral treatment of UP466 doses equivalent to as high as $22.68 \mathrm{~g} /$ day for average human showed no gastric mucosal or duodenal microscopic changes. These findings suggest that the unique blend of baicalin and catechin is well-tolerated by the gastrointestinal mucosa in rats and may presumably be well tolerated in humans. In this regard, UP446 is consistent with the reports of other flavonoids, for instance garcinol, rutin and quercetin, shown to either be gastro protective or have been used for the treatment of gastric ulcerations [23,29].

This study also showed that no adverse change in clinical chemistry parameters were observed in male or female rats treated with UP446. Even at the highest daily oral dosage of $2000 \mathrm{mg} / \mathrm{kg}$, UP446 did not induce any changes of liver enzymes such as ALT, AST, ALP and caused no changes of absolute and relative liver weight and no histopathology changes of liver tissues. The statistically significant changes in mean clinical chemistry for glucose in males administered $2000 \mathrm{mg} / \mathrm{kg} /$ day were not adverse or not related to expose to test substance because the differences in these parameters from those of the control groups were relatively small in magnitude they are not found in both sexes.

The absolute and relative organ weights in all treated groups except increased absolute adrenal gland weight at female recovery group were not significantly different from those of control group. Therefore, it was considered to be no toxicological significance since the organ did not show test substance related changes in the main group and these were secondary effects of body weight.

There were no macroscopic observations considered to be treatment related in this study. No gross abnormalities 
Table 9. Absolute (g) and relative organ weights (g/100g body weight) of male rats with UP446 26-week repeated oral dose toxicity with 4-week recovery period study.

\begin{tabular}{|c|c|c|c|c|c|c|}
\hline \multirow{2}{*}{ Parameters } & \multicolumn{4}{|c|}{ Main group (mg/kg) } & \multicolumn{2}{|c|}{ Recovery group (mg/kg) } \\
\hline & Control & UP446, 500 & UP446, 1000 & UP446, 2000 & Control & UP446, 2000 \\
\hline Body weight, $g$ & $729.9 \pm 83.1$ & $713.5 \pm 57.6$ & $715.3 \pm 83.4$ & $665.9 \pm 75.8$ & $735.7 \pm 123.9$ & $695.0 \pm 45.0$ \\
\hline $\begin{array}{c}\text { Brain, } \\
\mathrm{g}(\mathrm{g} / 100 \mathrm{~g} \mathrm{BW})\end{array}$ & $\begin{array}{c}2.19 \pm 0.13 \\
(0.30 \pm 0.03)\end{array}$ & $\begin{array}{c}2.19 \pm 0.10 \\
(0.31 \pm 0.03)\end{array}$ & $\begin{array}{c}2.18 \pm 0.08 \\
(0.31 \pm 0.04)\end{array}$ & $\begin{array}{c}2.20 \pm 0.09 \\
(0.33 \pm 0.04)\end{array}$ & $\begin{array}{c}2.20 \pm 0.06 \\
(0.30 \pm 0.04)\end{array}$ & $\begin{array}{c}2.13 \pm 0.08 \\
(0.31 \pm 0.02)\end{array}$ \\
\hline $\begin{array}{c}\text { Pituitary, } \\
\mathrm{g}(\mathrm{g} / 100 \mathrm{~g} \mathrm{BW})\end{array}$ & $\begin{array}{l}0.0148 \pm 0.0022 \\
0.0020 \pm 0.0003\end{array}$ & $\begin{array}{c}0.0154 \pm 0.0022 \\
(0.0022 \pm 0.0004)\end{array}$ & $\begin{array}{c}0.0144 \pm 0.0011 \\
(0.0020 \pm 0.0002)\end{array}$ & $\begin{array}{c}0.0154 \pm 0.0016 \\
(0.0024 \pm 0.0004)\end{array}$ & $\begin{array}{c}0.0148 \pm 0.0020 \\
(0.0020 \pm 0.0003)\end{array}$ & $\begin{array}{c}0.0157 \pm 0.0025 \\
(0.0022 \pm 0.0004)\end{array}$ \\
\hline $\begin{array}{c}\text { Thymus, } \\
\mathrm{g}(\mathrm{g} / 100 \mathrm{~g} \mathrm{BW})\end{array}$ & $\begin{array}{c}0.18 \pm 0.05 \\
(0.02 \pm 0.01)\end{array}$ & $\begin{array}{c}0.18 \pm 0.03 \\
(0.03 \pm 0.01)\end{array}$ & $\begin{array}{c}0.21 \pm 0.08 \\
(0.03 \pm 0.01)\end{array}$ & $\begin{array}{c}0.17 \pm 0.04 \\
(0.03 \pm 0.01)\end{array}$ & $\begin{array}{c}0.14 \pm 0.04 \\
(0.02 \pm 0.01)\end{array}$ & $\begin{array}{c}0.14 \pm 0.06 \\
(0.02 \pm 0.01)\end{array}$ \\
\hline $\begin{array}{c}\text { Heart, } \\
\mathrm{g}(\mathrm{g} / 100 \mathrm{~g} \mathrm{BW})\end{array}$ & $\begin{array}{c}1.77 \pm 0.21 \\
(0.24 \pm 0.01)\end{array}$ & $\begin{array}{c}1.77 \pm 0.17 \\
(0.25 \pm 0.02)\end{array}$ & $\begin{array}{c}1.75 \pm 0.20 \\
(0.24 \pm 0.02)\end{array}$ & $\begin{array}{c}1.65 \pm 0.16 \\
(0.25 \pm 0.02)\end{array}$ & $\begin{array}{c}1.87 \pm 0.18 \\
(0.26 \pm 0.03)\end{array}$ & $\begin{array}{c}1.78 \pm 0.12 \\
(0.26 \pm 0.01)\end{array}$ \\
\hline $\begin{array}{c}\text { Lung, } \\
\mathrm{g}(\mathrm{g} / 100 \mathrm{~g} \text { BW })\end{array}$ & $\begin{array}{c}1.75 \pm 0.18 \\
(0.24 \pm 0.01)\end{array}$ & $\begin{array}{c}1.77 \pm 0.07 \\
(0.25 \pm 0.02)\end{array}$ & $\begin{array}{c}1.69 \pm 0.13 \\
(0.24 \pm 0.02)\end{array}$ & $\begin{array}{c}1.67 \pm 0.18 \\
(0.25 \pm 0.02)\end{array}$ & $\begin{array}{c}1.84 \pm 0.18 \\
(0.25 \pm 0.03)\end{array}$ & $\begin{array}{c}1.75 \pm 0.09 \\
(0.25 \pm 0.02)\end{array}$ \\
\hline $\begin{array}{c}\text { Liver, } \\
\mathrm{g}(\mathrm{g} / 100 \mathrm{~g} \mathrm{BW})\end{array}$ & $\begin{array}{l}18.51 \pm 2.52 \\
(2.53 \pm 0.13)\end{array}$ & $\begin{array}{l}17.87 \pm 2.33 \\
(2.50 \pm 0.24)\end{array}$ & $\begin{array}{l}17.64 \pm 3.14 \\
(2.46 \pm 0.20)\end{array}$ & $\begin{array}{l}16.93 \pm 3.24 \\
(2.53 \pm 0.29)\end{array}$ & $\begin{array}{l}18.85 \pm 3.91 \\
(2.56 \pm 0.32)\end{array}$ & $\begin{array}{l}17.95 \pm 1.97 \\
(2.58 \pm 0.18)\end{array}$ \\
\hline $\begin{array}{c}\text { Spleen, } \\
\mathrm{g}(\mathrm{g} / 100 \mathrm{~g} \text { BW })\end{array}$ & $\begin{array}{c}1.06 \pm 0.13 \\
(0.15 \pm 0.02)\end{array}$ & $\begin{array}{c}1.05 \pm 0.26 \\
(0.15 \pm 0.03)\end{array}$ & $\begin{array}{c}0.98 \pm 0.29 \\
(0.14 \pm 0.03)\end{array}$ & $\begin{array}{c}0.89 \pm 0.11 \\
(0.13 \pm 0.01)\end{array}$ & $\begin{array}{c}0.92 \pm 0.08 \\
(0.13 \pm 0.02)\end{array}$ & $\begin{array}{c}0.96 \pm 0.15 \\
(0.14 \pm 0.02)\end{array}$ \\
\hline $\begin{array}{c}\text { Kidney, } \\
\mathrm{g}(\mathrm{g} / 100 \mathrm{~g} \text { BW })\end{array}$ & $\begin{array}{c}3.74 \pm 0.33 \\
(0.52 \pm 0.05)\end{array}$ & $\begin{array}{c}3.91 \pm 0.74 \\
(0.55 \pm 0.09)\end{array}$ & $\begin{array}{c}3.76 \pm 0.35 \\
(0.53 \pm 0.04)\end{array}$ & $\begin{array}{c}3.77 \pm 0.36 \\
(0.57 \pm 0.06)\end{array}$ & $\begin{array}{c}4.03 \pm 0.74 \\
(0.55 \pm 0.07)\end{array}$ & $\begin{array}{c}3.97 \pm 0.36 \\
(0.57 \pm 0.06)\end{array}$ \\
\hline $\begin{array}{c}\text { Adrenal, } \\
\mathrm{g}(\mathrm{g} / 100 \mathrm{~g} \mathrm{BW})\end{array}$ & $\begin{array}{c}0.0664 \pm 0.0094 \\
(0.0091 \pm 0.0008)\end{array}$ & $\begin{array}{c}0.0610 \pm 0.0085 \\
(0.0086 \pm 0.0011)\end{array}$ & $\begin{array}{c}0.0625 \pm 0.0182 \\
(0.0086 \pm 0.0017)\end{array}$ & $\begin{array}{c}0.0571 \pm 0.0112 \\
(0.0087 \pm 0.0023)\end{array}$ & $\begin{array}{c}0.0669 \pm 0.0088 \\
(0.0092 \pm 0.0013)\end{array}$ & $\begin{array}{c}0.0601 \pm 0.0137 \\
(0.0087 \pm 0.0020)\end{array}$ \\
\hline $\begin{array}{c}\text { Testis, } \\
\mathrm{g}(\mathrm{g} / 100 \mathrm{~g} \mathrm{BW})\end{array}$ & $\begin{array}{c}3.68 \pm 0.40 \\
(0.51 \pm 0.06)\end{array}$ & $\begin{array}{c}3.69 \pm 0.33 \\
(0.52 \pm 0.07)\end{array}$ & $\begin{array}{c}3.63 \pm 0.33 \\
(0.51 \pm 0.08)\end{array}$ & $\begin{array}{c}3.70 \pm 0.30 \\
(0.56 \pm 0.06)\end{array}$ & $\begin{array}{c}3.98 \pm 0.63 \\
(0.54 \pm 0.06)\end{array}$ & $\begin{array}{c}3.76 \pm 0.14 \\
(0.54 \pm 0.04)\end{array}$ \\
\hline $\begin{array}{c}\text { Prostate, } \\
\mathrm{g}(\mathrm{g} / 100 \mathrm{~g} \mathrm{BW})\end{array}$ & $\begin{array}{c}0.65 \pm 0.10 \\
(0.09 \pm 0.02)\end{array}$ & $\begin{array}{c}0.50 \pm 0.13 \\
(0.07 \pm 0.02)\end{array}$ & $\begin{array}{c}0.54 \pm 0.20 \\
(0.08 \pm 0.03)\end{array}$ & $\begin{array}{c}0.56 \pm 0.18 \\
(0.08 \pm 0.03)\end{array}$ & $\begin{array}{c}0.60 \pm 0.19 \\
(0.08 \pm 0.02)\end{array}$ & $\begin{array}{c}0.55 \pm 0.20 \\
(0.08 \pm 0.03)\end{array}$ \\
\hline
\end{tabular}

Mean hematological parameters were calculated and data were expressed as mean \pm SD $(n=10$, except control group; $n=9$, Recovery group; $n=5)$.

Table 10. Absolute (g) and relative organ weights (g/100g body weight) of female rats with UP446 26-week repeated oral dose toxicity with 4-week recovery period study.

\begin{tabular}{|c|c|c|c|c|c|c|}
\hline \multirow{2}{*}{ Parameters } & \multicolumn{4}{|c|}{ Main group (mg/kg) } & \multicolumn{2}{|c|}{ Recovery group (mg/kg) } \\
\hline & Control & UP446, 500 & UP446, 1000 & UP446, 2000 & Control & UP446, 2000 \\
\hline Body weight, g & $369.8 \pm 39.5$ & $348.9 \pm 48.7$ & $358.3 \pm 29.5$ & $355.2 \pm 42.4$ & $343.8 \pm 24.9$ & $358.8 \pm 39.0$ \\
\hline $\begin{array}{c}\text { Brain, g } \\
(\mathrm{g} / 100 \mathrm{~g} \mathrm{BW})\end{array}$ & $\begin{array}{c}1.94 \pm 0.08 \\
(0.53 \pm 0.05)\end{array}$ & $\begin{array}{c}1.89 \pm 0.14 \\
(0.55 \pm 0.07)\end{array}$ & $\begin{array}{c}1.94 \pm 0.08 \\
(0.55 \pm 0.05)\end{array}$ & $\begin{array}{c}1.95 \pm 0.10 \\
(0.55 \pm 0.06)\end{array}$ & $\begin{array}{c}1.95 \pm 0.09 \\
(0.57 \pm 0.03)\end{array}$ & $\begin{array}{c}1.99 \pm 0.07 \\
(0.56 \pm 0.04)\end{array}$ \\
\hline $\begin{array}{l}\text { Pituitary, g } \\
\text { (g/100g BW) }\end{array}$ & $\begin{array}{c}0.0200 \pm 0.0042 \\
(0.0055 \pm 0.0014)\end{array}$ & $\begin{array}{c}0.0214 \pm 0.0056 \\
(0.0062 \pm 0.0017)\end{array}$ & $\begin{array}{c}0.0197 \pm 0.0054 \\
(0.0055 \pm 0.0016)\end{array}$ & $\begin{array}{c}0.0231 \pm 0.0023 \\
(0.0066 \pm 0.0011)\end{array}$ & $\begin{array}{c}0.0201 \pm 0.0027 \\
(0.0059 \pm 0.0012)\end{array}$ & $\begin{array}{c}0.0230 \pm 0.0028 \\
(0.0065 \pm 0.0010)\end{array}$ \\
\hline $\begin{array}{l}\text { Thymus, g } \\
\text { (g/100g BW) }\end{array}$ & $\begin{array}{c}0.14 \pm 0.05 \\
(0.04 \pm 0.01)\end{array}$ & $\begin{array}{c}0.16 \pm 0.04 \\
(0.05 \pm 0.01)\end{array}$ & $\begin{array}{c}0.14 \pm 0.03 \\
(0.04 \pm 0.01)\end{array}$ & $\begin{array}{c}0.15 \pm 0.05 \\
(0.04 \pm 0.01)\end{array}$ & $\begin{array}{c}0.10 \pm 0.01 \\
(0.03 \pm 0.01)\end{array}$ & $\begin{array}{c}0.13 \pm 0.04 \\
(0.04 \pm 0.01)\end{array}$ \\
\hline $\begin{array}{c}\text { Heart, g } \\
\text { (g/100g BW) }\end{array}$ & $\begin{array}{c}1.07 \pm 0.07 \\
(0.29 \pm 0.03)\end{array}$ & $\begin{array}{c}1.06 \pm 0.12 \\
(0.31 \pm 0.03)\end{array}$ & $\begin{array}{c}1.08 \pm 0.08 \\
(0.30 \pm 0.02)\end{array}$ & $\begin{array}{c}1.08 \pm 0.09 \\
(0.31 \pm 0.02)\end{array}$ & $\begin{array}{c}1.05 \pm 0.09 \\
(0.30 \pm 0.03)\end{array}$ & $\begin{array}{c}1.09 \pm 0.12 \\
(0.30 \pm 0.04)\end{array}$ \\
\hline $\begin{array}{l}\text { Lung, g } \\
\text { (g/100g BW) }\end{array}$ & $\begin{array}{c}1.24 \pm 0.08 \\
(0.34 \pm 0.03)\end{array}$ & $\begin{array}{c}1.26 \pm 0.13 \\
(0.36 \pm 0.05)\end{array}$ & $\begin{array}{c}1.23 \pm 0.10 \\
(0.34 \pm 0.04)\end{array}$ & $\begin{array}{c}1.24 \pm 0.08 \\
(0.35 \pm 0.04)\end{array}$ & $\begin{array}{c}1.28 \pm 0.11 \\
(0.37 \pm 0.01)\end{array}$ & $\begin{array}{c}1.30 \pm 0.08 \\
(0.36 \pm 0.02)\end{array}$ \\
\hline $\begin{array}{c}\text { Liver, g } \\
\text { (g/100g BW) }\end{array}$ & $\begin{array}{c}8.68 \pm 0.76 \\
(2.36 \pm 0.24)\end{array}$ & $\begin{array}{c}8.58 \pm 1.28 \\
(2.48 \pm 0.38)\end{array}$ & $\begin{array}{c}8.85 \pm 0.95 \\
(2.47 \pm 0.17)\end{array}$ & $\begin{array}{c}9.04 \pm 1.06 \\
(2.55 \pm 0.21)\end{array}$ & $\begin{array}{c}8.59 \pm 0.72 \\
(2.50 \pm 0.13)\end{array}$ & $\begin{array}{c}9.07 \pm 1.07 \\
(2.53 \pm 0.23)\end{array}$ \\
\hline $\begin{array}{c}\text { Spleen, g } \\
(\mathrm{g} / 100 \mathrm{~g} \mathrm{BW})\end{array}$ & $\begin{array}{c}0.56 \pm 0.05 \\
(0.15 \pm 0.02)\end{array}$ & $\begin{array}{c}0.54 \pm 0.09 \\
(0.16 \pm 0.03)\end{array}$ & $\begin{array}{c}0.55 \pm 0.08 \\
(0.15 \pm 0.02)\end{array}$ & $\begin{array}{c}0.49 \pm 0.06 \\
(0.14 \pm 0.02)\end{array}$ & $\begin{array}{c}0.56 \pm 0.13 \\
(0.16 \pm 0.03)\end{array}$ & $\begin{array}{c}0.50 \pm 0.06 \\
(0.14 \pm 0.01)\end{array}$ \\
\hline $\begin{array}{l}\text { Kidney, g } \\
\text { (g/100g BW) }\end{array}$ & $\begin{array}{c}2.06 \pm 0.13 \\
(0.56 \pm 0.06)\end{array}$ & $\begin{array}{c}2.04 \pm 0.19 \\
(0.59 \pm 0.08)\end{array}$ & $\begin{array}{c}2.10 \pm 0.18 \\
(0.59 \pm 0.04)\end{array}$ & $\begin{array}{c}2.10 \pm 0.23 \\
(0.59 \pm 0.06)\end{array}$ & $\begin{array}{c}2.03 \pm 0.19 \\
(0.59 \pm 0.05)\end{array}$ & $\begin{array}{c}2.17 \pm 0.24 \\
(0.61 \pm 0.05)\end{array}$ \\
\hline $\begin{array}{l}\text { Adrenal, g } \\
(\mathrm{g} / 100 \mathrm{~g} \mathrm{BW})\end{array}$ & $\begin{array}{c}0.0708 \pm 0.0089 \\
(0.0192 \pm 0.0023)\end{array}$ & $\begin{array}{c}0.0668 \pm 0.0141 \\
(0.0194 \pm 0.0045)\end{array}$ & $\begin{array}{c}0.0638 \pm 0.0092 \\
(0.0178 \pm 0.0025)\end{array}$ & $\begin{array}{c}0.0762 \pm 0.0096 \\
(0.0217 \pm 0.0034)\end{array}$ & $\begin{array}{c}0.0677 \pm 0.0071 \\
(0.0198 \pm 0.0027)\end{array}$ & $\begin{array}{l}0.0079 \pm 0.0045^{*} \\
(0.0219 \pm 0.0028)\end{array}$ \\
\hline $\begin{array}{c}\text { Ovary, g } \\
(\mathrm{g} / 100 \mathrm{~g} \mathrm{BW})\end{array}$ & $\begin{array}{c}0.0712 \pm 0.0273 \\
(0.0194 \pm 0.0077)\end{array}$ & $\begin{array}{c}0.0638 \pm 0.0208 \\
(0.0181 \pm 0.0043)\end{array}$ & $\begin{array}{c}0.0690 \pm 0.0171 \\
(0.0194 \pm 0.0053)\end{array}$ & $\begin{array}{c}0.0674 \pm 0.0140 \\
(0.0191 \pm 0.0040)\end{array}$ & $\begin{array}{c}0.0767 \pm 0.0269 \\
(0.0221 \pm 0.0069)\end{array}$ & $\begin{array}{c}0.0687 \pm 0.0175 \\
(0.0189 \pm 0.0032)\end{array}$ \\
\hline $\begin{array}{c}\text { Uterus, } g \\
\text { (g/100g BW) }\end{array}$ & $\begin{array}{c}0.75 \pm 0.24 \\
(0.20 \pm 0.07)\end{array}$ & $\begin{array}{c}0.74 \pm 0.21 \\
(0.22 \pm 0.08)\end{array}$ & $\begin{array}{c}0.85 \pm 0.16 \\
(0.24 \pm 0.04)\end{array}$ & $\begin{array}{c}0.84 \pm 0.42 \\
(0.25 \pm 0.15)\end{array}$ & $\begin{array}{c}0.84 \pm 0.27 \\
(0.25 \pm 0.08)\end{array}$ & $\begin{array}{c}0.98 \pm 0.61 \\
(0.28 \pm 0.17)\end{array}$ \\
\hline
\end{tabular}

Mean hematological parameters were calculated and data were expressed as mean \pm SD $(n=10$, except control group; $n=9$, Recovery group; $n=5)$. Significantly different from control by Student t-test: ${ }^{*} p<0.05$. 
attributed to the test article were noted for any of the euthanized animals necropsied at the end of the 26-week observation period. Incidental findings correspond to historical control values for other experiments [30,31].

In the present study, the oral lethal dose of UP446 for male and female rats is in excess of $5000 \mathrm{mg} / \mathrm{kg}$ and the no observed adverse effect level (NOAEL) of the UP446 for both male and female rats is considered to be greater than $2000 \mathrm{mg} / \mathrm{kg} / \mathrm{day}$. Based on our present study, further toxicity study using beagle dogs with UP446 should be also conducted in GLP institute.

\section{Acknowledgements}

This study was funded by Chungcheong Leading Industry Promotion Project of the Korean Ministry of Knoweldge Economy. The authors would like to express their best gratitude to Dr. Edward Cannon, Dr. Doug Bradley, Dr. Wenwen Ma, Dr. Padma Abeysinghe, Dr. Min Chu and Unigen team for their incalculable support for the completion of this research.

\section{REFERENCES}

[1] M. Modak, P. Dixit, J. Londhe, S. Ghaskadbi and T. P. Devasagayam, "Indian Herbs and Herbal Drugs Used for Treatment of Diabetes," Journal of Clinical Biochemistry and Nutrition, Vol. 40, No. 3, 2007, pp. 163-173. doi: $10.3164 /$ jcbn. 40.163

[2] R. Morphy, C. Kay and Z. Rankovic, "From Magic Bullets to Designed Multiple Ligands," Drug Discovery Today, Vol. 9, No. 15, 2004, pp. 641-651. doi:10.1016/S1359-6446(04)03163-0

[3] P. A. Tatke, I. S. Nidhiya and S. G. Deshpande, "Safety Profile of Polyherbal Formulation in Female Rats by Subchronic Oral Ttoxicity Study," Toxicology International, Vol. 19, No. 2, 2012, pp. 106-111. doi:10.4103/0971-6580.97196

[4] P. G. Shekelle, S. C. Morton, M. J. Suttorp, N. Buscemi and C. Friesen, "Challenges in Systematic Reviews of Complementary and Alternative Medicine Topics," Annals of Internal Medicine, Vol. 142, No. 12, 2005, pp. 1042-1047.

doi:10.7326/0003-4819-142-12 Part 2-200506211-00003

[5] R. Kroes and R. Walker, "Safety Issues of Botanicals and Botanical Preparations in Functional Foods," Toxicology, Vol. 198, No. 1-3, 2004, pp. 213-220. doi:10.1016/j.tox.2004.01.028

[6] E. Middleton, C. Kandaswami and T. C. Theoharides, "The Effects of Plant Flavonoids on Mammalian Cells: Implications for Inflammation, Heart Disease, and Cancer," Pharmacological Review, Vol. 52, No. 4, 2000, pp. 673-751.

[7] M. M. Cowan, "Plant Products as Antimicrobial Agents," Clinical Microbiology Reviews, Vol. 12, No. 4, 1999, pp. 564-582.

[8] P. Knekt, J. Kumpulainen, R. Jarvinen, H. Rissanen, M.
Heliovaara, A. Reunanen, T. Hakulinen and A. Aromaa, "Flavonoid Intake and Risk of Chronic Diseases," The American Journal of Clinical Nutrition, Vol. 76, No. 3, 2002, pp. 560-568.

[9] L. Fu, B. T. Gan, Y. Zhang, X. R. Xu, E. Q. Xia and H. B. Li, "Total Phenolic Contents and Antioxidant Capacities of Herbal and Tea Infusions," International Journal of Molecular Sciences, Vol. 12, No. 4, 2011, pp. 2112-2124. doi:10.3390/ijms12042112

[10] Y. J. Moon, X. Wang and M. E. Morris, "Dietary Flavonoids: Effects on Xenobiotic and Carcinogen Metabolism," Toxicological in Vitro, Vol. 20, No. 2, 2006, pp. 187-210. doi:10.1016/j.tiv.2005.06.048

[11] T. G. Razina, S. N. Udintsev, I. I. Titutrin, T. G. Borovskaia and K. V. Iaremenko, "The Role of Thrombocyte Aggregation Function in the Mechanism of the Antimetastatic Action of an Extract of Baikal Skullcap," Voprosy Onkologii, Vol. 35, No. 3, 1989, pp. 331-335.

[12] N. Mahmood, C. Pizza, R. Aquino, N. De Tommasi, S. Piacente, S. Colman, A. Burke and A. J. Hay, "Inhibition of HIV Infection by Flavonoids," Antiviral Research, Vol. 22, No. 2-3, 1993, pp. 189-199. doi:10.1016/0166-3542(93)90095-Z

[13] I. M. Van Loon, "The golden Root: Rlinical Applications of Scutellaria Baicalensis GEORGI Flavonoids as Modulators of the Inflammatory Response," Alternative Medicine Review, Vol. 2, No. 6, 1992, pp. 472-480.

[14] B. H. Lee, S. J. Lee, T. H. Kang, D. H. Kim, D. H. Sohn, G. I. Ko and Y. C. Kim, "Baicalein: An in Vitro Antigenotoxic Compound from Scutellaria Baicalensis," Planta Medica, Vol. 66, No. 1, 2000, pp. 70-71. doi:10.1055/s-0029-1243111

[15] S. Shigeta, "Recent Progress in Antiviral Chemotherapy for Respiratory Syncytial Virus Infections," Expert Opinion on Investigational Drugs, Vol. 9, No. 2, 2000, pp. 221-235. doi:10.1517/13543784.9.2.221

[16] E. De Clercq, "Current Lead Natural Products for the Chemotherapy of Human Immunodeficiency Virus (HIV) Infection," Medicinal Research Reviews, Vol. 20, No. 5, 2000, pp. 323-349. doi:10.1002/1098-1128(200009)20:5<323::AID-MED1 $>3$ .0.CO;2-A

[17] Y. Huang, S. Y. Tsang, X. Yao and Z. Y. Chen, "Biological Properties of Baicalein in Cardiovascular System," Current Drug Targets Cardiovascular \& Haematological Disorder, Vol. 5, No. 2, 2005, pp. 177-184. doi: $10.2174 / 1568006043586206$

[18] B. P. Burnett, Q. Jia, Y. Zhao and R. M. Levy, “ A Medicinal Extract of Scutellaria baicalensis and Acacia catechu Acts as a Dual Inhibitor of Cyclooxygenase and 5Lipoxygenase to Reduce Inflammation," Journal of Medicinal Food, Vol. 10, No. 3, 2007, pp. 442-451. doi:10.1089/jmf.2006.255

[19] S. L. Morgan, J. E. Baggott, L. Moreland, R. Desmond and A. C. Kendrach, "The Safety of Flavocoxid, a Medical Food, in the Dietary Management of Knee Osteoarthritis," Journal of Medicinal Food, Vol. 12, No. 5, 2009, pp. 1143-1148. doi:10.1089/jmf.2008.0244 
[20] J. S. Sampalis and L. A. Brownell, "A Randomized, Double Blind, Placebo and Active Comparator Controlled Pilot Study of UP446, a Novel Dual Pathway Inhibitor Anti-Inflamatory Agent of Botanical Origin," Nutrition Journal, Vol. 11, 2012, p. 21.

[21] B. P. Burnett, S. Silva, M. H. Mesches, S. Wilson and Q. Jia, "Safety Evaluation of a Combination, Defined Extract of Scutellaria Baicalensis and Acacia Catechu," Journal of Food Biochemistry, Vol. 31, No. 6, 2007, pp. 797-825. doi:10.1111/j.1745-4514.2007.00142.x

[22] M. Yimam, L. Brownell, M. Hodges and Q. Jia, "Analgesic Effects of Standardized Bioflavonoid Composition from Scutellaria Baicalensis and Acacia Catechu," Journal of Dietary Supplements, Vol. 9, No. 3, 2012, pp. 155165. doi:10.3109/19390211.2012.708713

[23] M. Yimam, Y. Zhao, W. Ma, Q. Jia, S. G. Do and J. H. Shin, "90 Day Oral Toxicity Study of UP446, a Combination of Defined Extracts of Scutellaria Baicalensis and Acacia Catechu, in Rats," Food and Chemical Toxicology, Vol. 48, No. 5, 2012, pp. 1202-1209. doi:10.1016/j.fct.2010.02.011

[24] Q. Jia, "Formulation of a Mixture of Free-B-Ring Flavonoids and Flavans as a Therapeutic Agent," US Patent No. 7514469, 2009.

[25] A. A. Adeneyea, O. P. Ajagbonna, T. I. Adeleke and S. O. Bello, "Preliminary Toxicity and Phytochemical Studies of the Stem Bark Aqueous Extract of Musanga Cecropioides in Rats," Journal of Ethnopharmacology, Vol. 105, No. 3, 2006, pp. 374-379. doi:10.1016/j.jep.2005.11.027

[26] C. Bombardier, L. Laine, A. Reicin, D. Shapiro, R. Burgos-Vargas, B. Davis, R. Day, M. B. Ferraz, C. J. Haw- key, M. C. Hochberg, T. K. Kvien and T. J. Schnitzer, "Comparison of upper Gastrointestinal Toxicity of Rofecoxib and Naproxen in Patients with Rheumatoid Arthritis," The New England Journal of Medicine, Vol. 343, No. 21, 2000, pp. 1520-1528. doi:10.1056/NEJM200011233432103

[27] R. S. Bresalier, V. E. Friedewald Jr., R. E. Rakel, W. C. Roberts and G. W. Williams, "The Editor's Roundtable: Cyclooxygenase-2 Inhibitors and Cardiovascular Risk," The American Journal of Cardiology, Vol. 96, No. 11, 2005, pp. 1589-1604. doi:10.1016/j.amjcard.2005.09.069

[28] G. A. Elliott, A. Purmalis, D. A. VanderMeer and R. H. Denlinger, "The Propionic Acids. Gastrointestinal Toxicity in Various Species," Toxicologic Pathology, Vol. 16, No. 2, 1988, pp. 245-250. doi: $10.1177 / 019262338801600217$

[29] K. S. Mota, G. E. Dias, M. E. Pinto, A. Luiz-Ferreira, A R. Souza-Brito, C. A. Hiruma-Lima, J. M. Barbosa-Filho and L. M. Batista, "Flavonoids with Gastroprotective Activity," Molecules, Vol. 14, No. 3, 2009, pp. 979-1012. doi:10.3390/molecules 14030979

[30] A. A. Adeneyea, O. P. Ajagbonna, T. I. Adeleke and S. O. Bello, "Preliminary Ttoxicity and Phytochemical Studies of the Stem Bark Aqueous Extract of Musanga Cecropioides in Rats," Journal of Ethnopharmacology, Vol. 105, No. 3, 2006, pp. 374-379. doi:10.1016/j.jep.2005.11.027

[31] L. Johnson, C. S. Petty and W. B. Neaves, "A Comparative Study of Daily Sperm Production and Testicular Composition in Humans and Rats," Biology of Reproduction, Vol. 22, No. 5, 1980, pp. 1233-1243. 